\title{
The Relationship between Self-Perceived Burden and Posttraumatic Growth among Colorectal Cancer Patients: The Mediating Effects of Resilience
}

\author{
Chengshuai Zhang, ${ }^{1,2}$ Ruitong Gao, ${ }^{1}$ Jiandong Tai, ${ }^{3}$ Yuewei Li ${ }^{D},{ }^{1}$ Si Chen, ${ }^{3}$ Lei Chen, \\ Xiaobai Cao, ${ }^{1}$ Li Wang, ${ }^{1}$ Minghua Jia, ${ }^{1}$ and Feng Li ${ }^{1}{ }^{1}$ \\ ${ }^{1}$ School of Nursing, Jilin University, Changchun 130000, China \\ ${ }^{2}$ Shengli Oilfield Central Hospital, Dongying, China \\ ${ }^{3}$ The First Hospital of Jilin University, Changchun 130000, China
}

Correspondence should be addressed to Feng Li; fli@jlu.edu.cn

Received 27 April 2019; Accepted 24 June 2019; Published 12 September 2019

Academic Editor: Antonella Gigantesco

Copyright (c) 2019 Chengshuai Zhang et al. This is an open access article distributed under the Creative Commons Attribution License, which permits unrestricted use, distribution, and reproduction in any medium, provided the original work is properly cited.

At present, the influence factors of posttraumatic growth (PTG) in colorectal cancer (CRC) patients and the relationship between PTG, self-perceived burden (SPB), and resilience are not completely clear. Thus, the present study examined whether resilience and SPB could predict PTG in CRC patients. The role of resilience as a potential mediator was also assessed. Using a cross-sectional design, a convenience sample of 157 CRC patients was selected as subjects, from July to December 2016 in a third-grade hospital. It was found that the main influencing factors for the total PTG score of CRC patients included work status, affordability for medical expenses, and duration of illness. Resilience was positively correlated with PTG, SPB was negatively correlated with PTG, and resilience played an intermediary role. Our findings remind clinicians to treat the psychosocial response of CRC patients from multiple perspectives, with a focus on their positive aspects. By increasing resilience and reducing the patient's SPB, clinicians might enhance the patient's PTG and quality of life.

\section{Introduction}

Colorectal cancer (CRC) is the third most common global cancer and a leading cause of cancer-related death [1]. Over 1.2 million new CRC cases are reported each year, and 600,000 patients die of this disease. Due to changes in living standards and the dietary patterns of Chinese residents, the incidence of CRC has increased year by year and in some developed regions the reported number of cases exceed those of high incidence countries $[2,3]$.

Cancer patients have concerns over their survival time, treatment side effects, and recurrence. These problems increase cancer-related mental problems including anxiety or depressive symptoms, interpersonal stress, and loneliness $[4,5]$, but some studies suggest that most CRC patients experience positive changes [6-8]. Posttraumatic growth (PTG) describes the positive changes that individuals experience after stressful events $[9,10]$. Positive PTG can help cancer patients cope with cancer-related psychological stress, and so research on PTG would promote the adaptation, growth, and recovery of patients after the traumatic event of cancer.

$\mathrm{SPB}$, as a negative psychological experience, refers to the psychological feelings of guilt, frustration, and anxiety when patients believe they are becoming a burden to their family and others [11]. Studies have shown that cancer patients have high levels of SPB and PTG $[12,13]$.

Resilience, also known as resistance or psychological resilience, refers to the ability of individuals to successfully cope and adapt to difficulties and is one of the most commonly used indicators for evaluating positive psychological conditions $[14,15]$. As resilience represents a dynamic, changeable path that can raise the level of hope and positive attitude, so active interventions based on resilience might represent a favorable choice for cancer patients [16]. 
This study therefore proposes a correlation between SPB, resilience, and PTG. Resilience may represent a mediator of SPB that affects PTG. Hence, we aimed to provide a basis for clinical caregivers to intervene in CRC patients through understanding the relationship between the three variables and select better and more appropriate intervention opportunities and variables.

\section{Methods}

2.1. Subjects. A cross-sectional, observational study was conducted in a third-grade hospital in a northeast city in China from July to December 2016. Inclusion criteria were as follows: (1) age $\geq 18$ years old; (2) primary colorectal cancer diagnosed by pathology; (3) voluntary participation with informed consent. Exclusion criteria were as follows: (1) cognitive difficulties, difficulty in reading and completing questionnaires; (2) being in a critical condition without the ability to complete the study.

This study was approved by the ethics committee of the author's academy. All questionnaires were collected by trained research assistants. Prior to the investigation, patients were informed the purpose of the study, their voluntary participation, and the right to withdraw from the study at any stage. Participants signed informed consent forms prior to study initiation and filled out the questionnaire during their hospitalization.

\subsection{Research Tools}

2.2.1. General Information Questionnaire. The general information questionnaire consisted of 13 items, including age, gender, marital status, and education level.

2.2.2. Posttraumatic Growth Inventory-Chinese Version. The Posttraumatic Growth Inventory (PTGI) [14] was used to assess the degree of positive psychological changes after the individual has experienced a traumatic event, including five dimensions: (1) relating to others, (2) new possibilities, (3) personal strength, (4) spiritual change, and (5) appreciation of life, totaling 21 items. We applied the Chinese revised version of PTGI (C-PTGI) [17]. The Likert 6 used by the C-PTGI, ranged from "no posttraumatic changes" (0 points) to "very large posttraumatic changes" (5 points), with a total score of 0 to 100 points. A higher score indicated a more positive psychological experience. In this study, Cronbach's coefficient of the CPTGI was 0.918, indicating a good reliability and validity.

2.2.3. Self-Perceived Burden Scale. The self-perceived burden scale (SPBS) included three dimensions of body burden, emotional burden, and economic burden, consisting of 10 items [18]. Cronbach's $\alpha$ score was 0.85 [19]. The SPBS score adopted a Likert 5 rating, from "never" (1 point) to "always" (5 points), with a total score that was either positive or negative (only the eighth item was scored in reverse, the others were positive scores). A higher total score indicates a higher level of individual SPB.

2.2.4. Connor-Davidson Resilience Scale. The ConnorDavidson Resilience Scale (CD-RISC) consisted of three dimensions: tenacity, strength, and optimism, totaling 25 entries. Cronbach's $\alpha$ coefficient was 0.93 [20].

2.3. Statistical Analysis. We used the Epidata 3.1 to establish a database and SPSS 21.0 for statistical analysis. The general information of CRC patients was analyzed by descriptive statistical analysis of frequency and percentage. The status of PTG was statistically described by the mean and standard deviation. Univariate analysis and multiple stepwise regression were used to analyze the influencing factors of PTG. A Pearson or Spearman correlation analysis was performed to assess the relationship between PTG and SPB. The structural equation model was constructed using AMOS.

\section{Results}

3.1. Participant General Information. Considering the number of entries in C-PTGI used in this survey was 21 and the sample loss of $10 \% \sim 20 \%$, in order to ensure sufficient sample size, a total of 200 questionnaires were distributed [21]. Finally, 190 questionnaires were collected, and incomplete questionnaires or all entries with the same score were considered invalid and excluded.

Overall, 157 questionnaires were accepted and the questionnaire response efficiency was 78.5\%. Among these, $49 \%$ of participants were over 60 years old, more males $(58.6 \%)$. The vast majority of participants were married (95.5\%) and currently lived with their spouses (75.2\%). CRC treatments were mainly surgery with radiotherapy or chemotherapy $(70.7 \%)$, and the duration of illness lasted from one month to one year $(70.1 \%)$. Specific data is shown in Table 1 .

3.2. Scores of PTG, SPB, and Resilience. The mean score of PTG was 76.78 ( $\mathrm{SD}=14.98$; range 22-100), with a mean score per item of 3.84 (on a 0-5 scale), which reflected an upper-moderate level of PTG among the CRC patients. The mean score of $\mathrm{SPB}$ was $34.81(\mathrm{SD}=6.82$; range 19 46 ), with a mean score per item of 3.48 (on a 1-5 scale), which reflected a moderate level of SPB among the CRC patients. The mean score of resilience was 69.03 ( $\mathrm{SD}=19.06$; range 18-100), with a mean score per item of 2.761 (on a 0 4 scale), which reflected a relatively low level of resilience among the CRC patients. Means and standard deviation of all variables regarding PTG, SPB, and resilience are shown in Table 2 .

3.3. Analysis of the Factors Influencing PTG in CRC Patients. The results revealed significant differences $(P<0.05)$ in age, work status, economic affordability, therapy method, illness time, and living arrangements. Table 1 shows specific data. The results of multivariate analysis showed that work status, 
TABLE 1: Participants' characteristics and differences of the PTG in different groups $(\mathrm{N}=196)$.

\begin{tabular}{|c|c|c|c|c|c|}
\hline Characteristic & Category & $\mathrm{N}(\%)$ & Mean \pm SD & $F / t / z / \chi^{2}$ & $P$ \\
\hline \multirow{4}{*}{ Age } & $18 \sim 40$ & $17(10.8)$ & $78.12 \pm 16.85$ & \multirow{4}{*}{$8.753^{\mathrm{a}}$} & \multirow{4}{*}{$0.033 *$} \\
\hline & $41 \sim 50$ & $30(19.1)$ & $71.77 \pm 13.30$ & & \\
\hline & $51 \sim 60$ & $33(21.0)$ & $79.67 \pm 19.58$ & & \\
\hline & $>60$ & $77(49.0)$ & $77.21 \pm 12.56$ & & \\
\hline \multirow{2}{*}{ Gender } & Male & $92(58.6)$ & $76.57 \pm 15.07$ & \multirow{2}{*}{-0.217} & \multirow{2}{*}{0.829} \\
\hline & Female & $65(41.4)$ & $77.09 \pm 14.96$ & & \\
\hline \multirow[t]{4}{*}{ Marital status } & Married & 128 & $77.26 \pm 15.17$ & \multirow{3}{*}{$4.333^{\mathrm{a}}$} & \multirow{3}{*}{0.115} \\
\hline & Single & 7 & $66.80 \pm 3.35$ & & \\
\hline & Divorced/widowed & 22 & $74.14 \pm 14.28$ & & \\
\hline & Retirement & $66(42.0)$ & $82.56 \pm 11.17$ & \multirow{4}{*}{$18.902^{\mathrm{a}}$} & \multirow{4}{*}{$\leq 0.001 * *$} \\
\hline \multirow{3}{*}{ Work status } & On job & $43(27.4)$ & $73.65 \pm 19.71$ & & \\
\hline & Others & $48(30.6)$ & $71.65 \pm 11.92$ & & \\
\hline & (Dimission/suspension) & $10(70.0)$ & 1.0011.0 & & \\
\hline \multirow{2}{*}{ Education } & Less than high school & $80(51.0)$ & $78.86 \pm 12.52$ & \multirow{2}{*}{1.775} & \multirow{2}{*}{0.078} \\
\hline & High school and above & $77(49.0)$ & $74.62 \pm 16.98$ & & \\
\hline \multirow{2}{*}{ Medical expenses payment method } & Medical insurance & 143(91.1) & $76.14 \pm 11.90$ & \multirow{2}{*}{-0.205} & \multirow{2}{*}{0.840} \\
\hline & Self-paying & $14(8.9)$ & $76.85 \pm 15.28$ & & \\
\hline \multirow[t]{2}{*}{ Residence } & Urban & $98(62.4)$ & $77.14 \pm 15.82$ & \multirow{2}{*}{1.037} & \multirow{2}{*}{0.301} \\
\hline & Rural & $59(37.8)$ & $75.19 \pm 13.45$ & & \\
\hline \multirow{2}{*}{$\begin{array}{l}\text { Affordability for } \\
\text { medical expenses }\end{array}$} & Yes & $119(75.8)$ & $79.32 \pm 15.55$ & \multirow{2}{*}{$5.495^{\mathrm{a}}$} & \multirow{2}{*}{$\leq 0.001 * *$} \\
\hline & No & $38(24.2)$ & $68.25 \pm 8.62$ & & \\
\hline \multirow[t]{3}{*}{ Religion } & Yes & $8(5.1)$ & $77.25 \pm 15.88$ & \multirow[t]{3}{*}{0.090} & \multirow[t]{3}{*}{0.928} \\
\hline & No & $149(94.9)$ & $76.76 \pm 14.99$ & & \\
\hline & $<¥ 1000$ & $27(17.2)$ & $75.22 \pm 13.19$ & & \\
\hline \multirow[t]{3}{*}{ Income (RMB)/month } & $¥ 1000 \sim 5000$ & $101(64.3)$ & $77.31 \pm 14.27$ & 0.215 & 0.807 \\
\hline & $>¥ 5000$ & $29(18.5)$ & $76.41 \pm 18.91$ & & \\
\hline & Radiation+ Chemotherapy & $32(20.4)$ & $75.06 \pm 14.63$ & & \\
\hline Therapy method & Surgery+ Radiation/Chemotherapy & $111(70.7)$ & $78.94 \pm 13.98$ & 7.290 & $0.001 * *$ \\
\hline & Surgery + Radiation + Chemotherapy & $14(8.9)$ & $63.64 \pm 17.18$ & & \\
\hline & $\leq 1$ month & $30(19.1)$ & $80.40 \pm 13.72$ & & \\
\hline Duration of illness & 1 month $\sim 1$ year & $110(70.1)$ & $77.25 \pm 14.63$ & 4.442 & $0.013 * *$ \\
\hline & $>1$ year & $17(10.8)$ & $67.41 \pm 16.37$ & & \\
\hline & Live alone & $10(6.4)$ & $66.50 \pm 14.63$ & & \\
\hline Living arrangement & Live with spouse & $118(75.2)$ & $78.21 \pm 15.38$ & 3.321 & $0.039 * *$ \\
\hline & Live with children & $29(18.5)$ & $74.52 \pm 11.90$ & & \\
\hline
\end{tabular}

$* P<0.05 ; * * P<0.01$, statistically significant.

TABLE 2: Means, standard deviations, and correlations among PTG, SPB, and resilience $(\mathrm{N}=196)$.

\begin{tabular}{lcccc}
\hline Variable & Mean \pm SD & Range & 1 & 2 \\
\hline 1. PTG & $76.78 \pm 14.98$ & $22 \sim 100$ & 1 & $-0.21 * *$ \\
2. SPB & $34.81 \pm 6.82$ & $19 \sim 46$ & & 1 \\
3. Resilience & $69.03 \pm 19.06$ & $18 \sim 100$ & & $-0.337 * *$ \\
\hline
\end{tabular}

$* * P<0.01$, statistically significant.

economic affordability, and duration of illness were the main factors affecting PTG in CRC patients (Table 3 ).

3.4. Correlations among PTG, SPB, and Resilience in CRC Patients. The results showed that the total PTG scores were negatively correlated with the total SPB scores $(r=-0.21$,
$P<0.01)$ and other dimensions were negatively correlated excluding body burden and the appreciation of life. The total and each dimension scores of SPB were negatively correlated with resilience. There is a positive correlation between PTG and resilience $(r=0.73, P<0.01)$. Specific results are shown in Table 2. 
TABLE 3: Multivariate analysis of PTG in colorectal cancer patients.

\begin{tabular}{|c|c|c|c|c|}
\hline Variable & $B$ & Beta & $t$ & $P$ \\
\hline Constant & 113.611 & & 12.675 & $\leq 0.001$ \\
\hline Work status & -4.350 & -0.246 & -3.130 & 0.002 \\
\hline Duration of illness & -6.257 & -0.227 & -3.081 & 0.002 \\
\hline Affordability for medical expenses & -8.953 & -0.252 & -3.187 & 0.002 \\
\hline
\end{tabular}

$R^{2}=0.216$, adjusted $R^{2}=0.185$, and $F=6.901$.

TABLE 4: Regression analysis of SPB on PTG.

\begin{tabular}{lcccc}
\hline Variable & $B$ & Beta & $t$ & $P$ \\
\hline Constant & 92.836 & & 15.171 & $\leq 0.001$ \\
SPB & -0.461 & -0.210 & -2.673 & 0.008 \\
\hline
\end{tabular}

$R^{2}=0.044$, adjusted $R^{2}=0.038$, and $F=7.143$.

TABLE 5: Regression analysis of SPB on resilience.

\begin{tabular}{lcccc}
\hline Variable & $B$ & Beta & $t$ & \multicolumn{1}{c}{ P } \\
\hline Constant & 101.780 & & 13.571 & $\leq 0.001$ \\
SPB & -0.941 & -0.337 & -4.449 & $\leq 0.001$ \\
\hline
\end{tabular}

$R^{2}=0.113$, adjusted $R^{2}=0.108$, and $F=19.795$.

TABLE 6: Regression analysis of resilience on PTG.

\begin{tabular}{lcccc}
\hline Variable & $B$ & Beta & $t$ & $P$ \\
\hline Constant & 37.198 & & 12.036 & $\leq 0.001$ \\
Resilience & 0.573 & 0.730 & 13.285 & $\leq 0.001$ \\
\hline
\end{tabular}

$R^{2}=0.532$, adjusted $R^{2}=0.529$, and $F=176.495$.

TABLE 7: Regression analysis of SPB and resilience on PTG.

\begin{tabular}{lcccc}
\hline Variable & $B$ & Beta & $t$ & \multicolumn{1}{c}{ 作 } \\
\hline Constant & 33.389 & & 5.265 & $\leq 0.001$ \\
Resilience & 0.584 & 0.743 & 12.721 & $\leq 0.001$ \\
SPB & 0.088 & 0.040 & 0.688 & 0.492 \\
\hline
\end{tabular}

$R^{2}=0.534$, adjusted $R^{2}=0.528$, and $F=88.185$.

\subsection{Path Analysis of SPB in the Process of PTG Affected by Resilience}

3.5.1. Regression Analysis of SPB on PTG. The results of regression analysis showed $R^{2}$ of $4 \%$, indicating that SPB could explain the variance of $4 \%$ in the PTG. The specific results are shown in Table 4.

3.5.2. Regression Analysis of SPB on Resilience. The results of regression analysis showed $R^{2}$ of $11.3 \%$, indicating that SPB could explain the variance of $11.3 \%$ in the resilience. The specific results are shown in Table 5.

3.5.3. Regression Analysis of Resilience on PTG. The results of regression analysis showed $R^{2}$ of $53.2 \%$, indicating that resilience could explain the variance of $53.2 \%$ in the PTG. The specific results are shown in Table 6.
3.5.4. Regression Analysis of SPB and Resilience on PTG. The results of regression analysis showed $R^{2}$ of $53.4 \%$, indicating that SPB and resilience could explain the variance of $53.4 \%$ in the PTG. At the same time, the SPB showed no effect on PTG. The specific results are shown in Table 7.

The SPB was found to influence the PTG level of CRC patients by affecting the level of resilience. The standardized path coefficient was $\mathrm{c}=-0.210(P=0.008)$. From regression analysis, the impact of SPB on resilience using the standardized regression of coefficient $\mathrm{a}=-0.337(P \leq 0.001)$ showed $\mathrm{a}$ significant difference. From the regression analysis of the impact of resilience on PTG, the path coefficient of $b=0.730$ $(P \leq 0.001)$ reached a significant level, and thus resilience affected PTG. The SPB and resilience were simultaneously included when calculating the regression of PTG. The path coefficient of $c^{\prime}=0.040$ did not show a significance influence $(P=0.492)$, indicating that the effects of SPB on PTG were 
TABLE 8: Path analysis of SPB in the process of PTG affected by resilience.

\begin{tabular}{lcc}
\hline & Standardized regression equation & Regression coefficient test \\
\hline Step 1 & $\mathrm{Y}=-0.210$ & $\mathrm{SE}=0.173, \mathrm{t}=-2.673 * *$ \\
Step 2 & $\mathrm{M}=-0.337$ & $\mathrm{SE}=0.211, \mathrm{t}=-4.449 * *$ \\
Step 3 & $\mathrm{Y}=0.040+0.743 \mathrm{M}$ & $\mathrm{SE}=0.128, \mathrm{t}=0.688$ \\
& & $\mathrm{SE}=0.046, \mathrm{t}=12.721 * *$ \\
\hline
\end{tabular}

$* * \mathrm{P}<0.01$

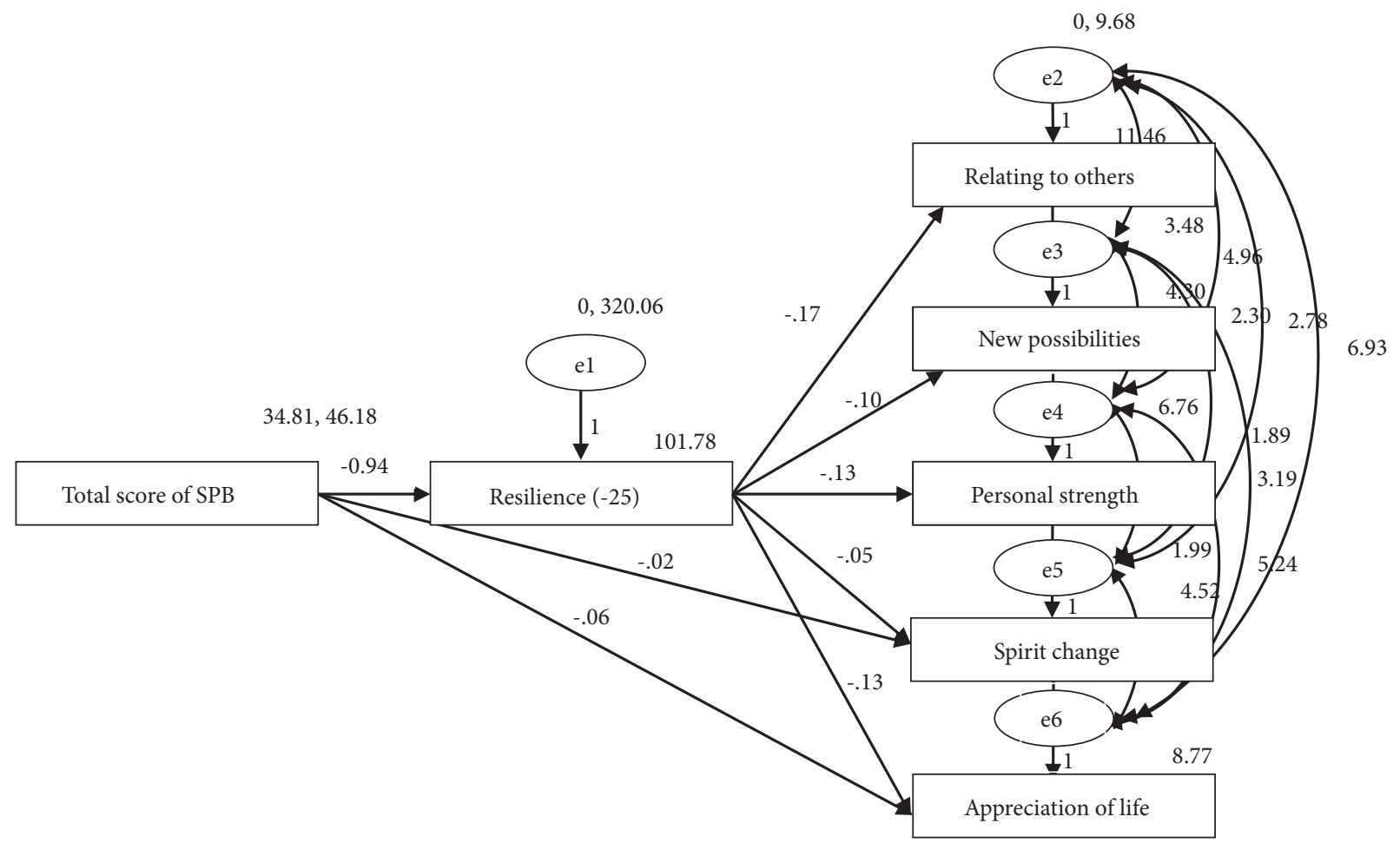

FIGURE 1: Initial model fitting and model modification.

through resilience. Resilience therefore played an intermediary role in the process of SPB affecting PTG (Table 8).

3.6. Path Effect Decomposition. We constructed and fitted the initial theoretical model which was amended using the model correction index (MI). The revised model is shown in Figure 1. The fit results showed that $x^{2} / d f=0.48<2(P=0.315)$, other indicators such as GFI, AGFI, NFI, TLI, CFI, and IFI were greater than 0.95 , and RMSEA $<0.05$, indicating that the model had a good fit and was more reasonable (Table 9).

By decomposing the path coefficients, we found that the SPB had no direct effect on the other dimensions of PTG, except for the spiritual change. The results showed that the effects of the SPB to PTG were through resilience. We observed a direct influencing spiritual change and the appreciation of life, but the direct action path coefficient of the appreciation of life dimension was not significant $(P=0.10)$, indicating that the influence was through the indirect effects of resilience. The effects of SPB on each dimension of PTG were spiritual change -0.260 , new possibilities -0.231 , appreciation of life -0.112 , relating to others -0.244 , and personal power -0.240 (Table 10).

\section{Discussion}

This study aims to explore the relationship between PTG, SPB and resilience in colorectal cancer patients. The results showed that PTG was negatively correlated with SPB and positively correlated with resilience. In addition, resilience played a full mediating role in PTG and SPB in colorectal cancer patients.

The PTG scores in this study were higher than scores in lung cancer, breast cancer, and colorectal cancer survivors with permanent intestinal ostomies [22-24]. This may be due to the continuous improvement of the cure rate and survival rate of colorectal cancer, which might influence their perception of positive findings. Moreover, none of the colorectal cancer survivors in our study underwent colostomy; after all, ostomy is a permanent injury for patients, which inevitably affects body function and changes body image, leading to more negative emotions. 
TABLE 9: Path model fitting index.

\begin{tabular}{lcccccccc}
\hline & CMIN/DF & GFI & AGFI & NFI & TLI & CFI & IFI & RMSEA \\
\hline This model & 1.183 & 0.994 & 0.988 & 0.996 & 0.996 & 0.999 & 0.999 & 0.034 \\
Standard & $<2$ & $>0.95$ & $>0.95$ & $>0.95$ & $>0.95$ & $>0.95$ & $>0.95$ & $<0.05$ \\
\hline
\end{tabular}

TABLE 10: Model path effect decomposition.

\begin{tabular}{lcccc}
\hline Effect & Independent variable & Standardized direct effect & Standardized indirect effect & Total standardized effect \\
\hline Resilience & Total SPB scores & -0.337 & 0.000 & -0.337 \\
Spiritual change & Resilience & 0.519 & 0.000 & 0.519 \\
New possibilities & Resilience & 0.686 & 0.000 & 0.686 \\
Appreciation of life & Resilience & 0.615 & 0.000 & 0.615 \\
Relating to others & Resilience & 0.726 & 0.000 & 0.726 \\
Personal strength & Resilience & 0.713 & 0.000 & 0.713 \\
Spiritual change & Total SPB scores & -0.86 & -0.175 & -0.260 \\
New possibilities & Total SPB scores & 0.000 & -0.231 & -0.231 \\
Appreciation of life & Total SPB scores & 0.095 & -0.207 & -0.112 \\
Relating to others & Total SPB scores & 0.000 & -0.244 & -0.244 \\
Personal strength & Total SPB scores & 0.000 & -0.240 & -0.240 \\
\hline
\end{tabular}

Multivariate analysis showed that PTG was associated with work status, affordability for medical expenses, and duration of illness. Regard work status, in current study we found the retirees have the highest PTG, in line with the research of Wang et al. [25]. Firstly, retirees are older; their understanding of life and themselves may be more comprehensive and profound, the more mature and effective coping styles which may lead to more positive psychological changes [26]. Secondly, the retirees have relatively free time, so they would spend more time in receiving treatment [27]. Thirdly, the retired people do not have to deal with the pressure and burden from work and have less concern about the impact of the disease on future work. Consistent with previous studies, this study also found that individuals who can afford medical expenses have higher posttraumatic growth levels $[25,28,29]$. This may be because they have less concern about economic problems and more resources [30]; they can afford more expensive drugs or treatment. Patients feel less burden, negative emotions, and disease uncertainty, thus promoting their PTG. Patients with shorter duration of illness in this study have higher PTG. It may be because, with the prolonged illness, the patient suffers from the pain, gradually loses confidence, and becomes more worried about the treatment or prognosis. At the same time, high medical expenses and the use of social resources also lead to the increase of patients' SPB to some extent, resulting in sense of guilt and negative emotions, which further leads to the decline of PTG. However, in some studies of breast cancer patients, higher PTG was found in patients with longer disease duration [31, 32]. This may be caused by disease specificity.

Previous studies have shown a correlation between PTG and negative psychological status. The level of PTG was significantly correlated with the painful condition of head and neck cancer and breast cancer patients and was significantly negatively correlated with depression and anxiety $[33,34]$. Perceived stress was also negatively associated with PTG $(r=0.36, P<0.001)$ [35]. Consistent with previous studies, SPB, as a negative psychological experience, was negatively correlated with PTG in colorectal cancer patients.

In keeping with previous studies, this study also found individuals with better resilience experience would have a higher degree of growth after traumatic events [24, 36, 37]. Resilient individuals are more likely to appraise adverse situations as challenges and exhibit cognitive flexibility, which contribute to the positive changes.

This study demonstrates that the effect of SPB on PTG is entirely through the resilience, which is consistent with the findings of the role of resilience in PTG and ruminating [36]. The mediating effect of resilience provides a new perspective for improving PTG in colorectal cancer patients. Resilience as a factor that can be adjusted in multiple ways, for example, from social-emotional training and training in cognitive reappraisal, clinicians should use it appropriately to improve health outcomes [38-40].

This study has several limitations. Firstly, it was a crosssectional study, a causal relationship cannot be inferred, and it cannot provide information on dynamic changes of PTG after colorectal cancer. Secondly, the sample size in this study was relatively small, only limited to the third-grade hospitals in a city in China. Thirdly, the effect of the treatment method on patients' psychology was not considered, such as the side effects of radiotherapy and chemotherapy. Therefore, the results had specific limitations. In future work, it is necessary to expand the sample size and scope, perform follow-up reports from the initial diagnosis of cancer patients, analyze dynamic changes of PTG levels in various periods, and 
comprehensively integrate the relevant factors of PTG to form a high level theoretical framework.

\section{Conclusion}

On the above, there is a correlation between $\mathrm{SPB}$, resilience, and PTG in colorectal cancer patients; the level of SPB and resilience has a certain predictive effect on PTG. Resilience plays a fully intermediary role in the impact of SPB on PTG. In the future, it is necessary to study the methods to improve PTG and resilience in colorectal cancer patients.

\section{Data Availability}

The data used to support the findings of this study are available from the corresponding author upon request.

\section{Conflicts of Interest}

None of the authors has any potential financial conflicts of interest related to this manuscript.

\section{Authors' Contributions}

Chengshuai Zhang and Ruitong Gao have contributed equally to this work.

\section{Acknowledgments}

This work was supported by the National Natural Science Foundation of China [grant number 81570491].

\section{References}

[1] R. L. Siegel, K. D. Miller, S. A. Fedewa et al., "Colorectal cancer statistics," CA: A Cancer Journal for Clinicians, vol. 67, no. 3, pp. 177-193, 2017.

[2] H. Brenner, M. Kloor, and C. P. Pox, "Toward better control of colorectal cancer," The Lancet, vol. 383, no. 9927, pp. 1490-1502, 2014.

[3] D. Wen, W. Zou, X. Wen et al., "Urban-rural disparity in colorectal cancer incidence and increasing trend in relation to socioeconomic development and urbanization in China," Journal of International Medical Research, vol. 46, no. 10, pp. 4181-4196, 2018.

[4] G. T. Deimling, K. F. Bowman, S. Sterns, L. J. Wagner, and B. Kahana, "Cancer-related health worries and psychological distress among older adult, long-term cancer survivors," PsychoOncology, vol. 15, no. 4, pp. 306-320, 2006.

[5] G. T. Deimling, B. Kahana, K. F. Bowman, and M. L. Schaefer, "Cancer survivorship and psychological distress in later life," Psycho-Oncology, vol. 11, no. 6, pp. 479-494, 2002.

[6] A. L. Hawkes, K. I. Pakenham, S. K. Chambers, T. A. Patrao, and K. S. Courneya, "Effects of a Multiple Health Behavior Change Intervention for Colorectal Cancer Survivors on Psychosocial Outcomes and Quality of Life: a Randomized Controlled Trial," Annals of Behavioral Medicine, vol. 48, no. 3, pp. 359-370, 2014.

[7] S. Occhipinti, S. K. Chambers, S. Lepore, J. Aitken, J. Dunn, and J. D. Elhai, "A Longitudinal Study of Post-Traumatic Growth and
Psychological Distress in Colorectal Cancer Survivors," PLoS ONE, vol. 10, no. 9, p. e0139119, 2015.

[8] L. Jansen, M. Hoffmeister, J. Chang-Claude, H. Brenner, and V. Arndt, "Benefit finding and post-traumatic growth in longterm colorectal cancer survivors: prevalence, determinants, and associations with quality of life," British Journal of Cancer, vol. 105, no. 8, pp. 1158-1165, 2011.

[9] M. Pérez-San-Gregorio, A. Martín-Rodríguez, M. Borda-Mas, M. Avargues-Navarro, J. Pérez-Bernal, and M. Gómez-Bravo, "Family Caregivers of Liver Transplant Recipients: Coping Strategies Associated With Different Levels of Post-traumatic Growth," Transplantation Proceedings, vol. 50, no. 2, pp. 646649, 2018.

[10] R. G. Tedeschi and L. G. Calhoun, "The posttraumatic growth inventory: measuring the positive legacy of trauma," Journal of Traumatic Stress, vol. 9, no. 3, pp. 455-471, 1996.

[11] T. Akazawa, T. Akechi, T. Morita et al., "Self-Perceived Burden in Terminally Ill Cancer Patients: A Categorization of Care Strategies Based on Bereaved Family Members' Perspectives," Journal of Pain and Symptom Management, vol. 40, no. 2, pp. 224-234, 2010.

[12] C. J. Mcpherson, K. G. Wilson, M. M. Lobchuk, and S. Brajtman, "Self-Perceived Burden to Others: Patient and Family Caregiver Correlates," Journal of Palliative Care, vol. 23, no. 3, pp. 135-142, 2019.

[13] Y. Yu, L. Peng, L. Chen et al., "Resilience and social support promote posttraumatic growth of women with infertility: The mediating role of positive coping," Psychiatry Research, vol. 215, no. 2, pp. 401-405, 2014.

[14] S. L. Szanton and J. M. Gill, "Facilitating Resilience Using a Society-to-Cells Framework," Advances in Nursing Science, vol. 33, no. 4, pp. 329-343, 2010.

[15] R. Tillery, K. M. Howard Sharp, Y. Okado, A. Long, and S. Phipps, "Profiles of Resilience and Growth in Youth with Cancer and Healthy Comparisons," Journal of Pediatric Psychology, vol. 41, no. 3, pp. 290-297, 2016.

[16] C. J. McPherson, K. G. Wilson, and M. A. Murray, "Feeling like a burden: Exploring the perspectives of patients at the end of life," Social Science \& Medicine, vol. 64, no. 2, pp. 417-427, 2007.

[17] Y. Wang, H. Wang, J. Wang, J. Wu, and X. Liu, "Prevalence and Predictors of Posttraumatic Growth in Accidentally Injured Patients," Journal of Clinical Psychology in Medical Settings, vol. 20, no. 1, pp. 3-12, 2013.

[18] L. A. Simmons, "Self-perceived Burden in Cancer Patients," Cancer Nursing, vol. 30, no. 5, pp. 405-411, 2007.

[19] N. Cousineau, I. McDowell, S. Hotz, and P. Hébert, "Measuring chronic patients' feelings of being a burden to their caregivers development and preliminary validation of a scale," Medical Care, vol. 41, no. 1, pp. 110-118, 2003.

[20] J. Tian and J. S. Hong, "Validation of the Chinese version of the Resilience Scale and its cutoff score for detecting low resilience in Chinese cancer patients," Supportive Care in Cancer, vol. 21, no. 5, pp. 1497-1502, 2013.

[21] M. Kendall, Multivariate analysis, London, UK, Charles Griffin, 1975.

[22] X. Peng, Y. Su, W. Huang, and X. Hu, "Status and factors related to posttraumatic growth in patients with lung cancer," Medicine, vol. 98, no. 7, p. e14314, 2019.

[23] N. Koutrouli, F. Anagnostopoulos, F. Griva et al., "Exploring the relationship between posttraumatic growth, cognitive processing, psychological distress, and social constraints in a sample 
of breast cancer patients," Women \& Health, vol. 56, no. 6, pp. 650-667, 2015.

[24] X. Dong, G. Li, C. Liu et al., "The mediating role of resilience in the relationship between social support and posttraumatic growth among colorectal cancer survivors with permanent intestinal ostomies: A structural equation model analysis," European Journal of Oncology Nursing, vol. 29, pp. 47-52, 2017.

[25] M. Wang, J. Liu, H. Wang, J. Chen, and Y. Li, "Posttraumatic growth and associated socio-demographic and clinical factors in Chinese breast cancer survivors," European Journal of Oncology Nursing, vol. 18, no. 5, pp. 478-483, 2014.

[26] Ruiz and Pedro, "Resilience and Mental Health," The Journal of Nervous and Mental Disease, vol. 200, no. 8, p. 734, 2012.

[27] M. M. Skeels, K. T. Unruh, C. Powell, and W. Pratt, "Catalyzing social support for breast cancer patients," in Proceedings of the SIGCHI Conference on Human Factors in Computing Systems, pp. 173-182, 2010.

[28] H. Yorulmaz, S. Bayraktar, and K. Özdilli, "Posttraumatic growth in chronic kidney failure disease," Procedia - Social and Behavioral Sciences, vol. 5, pp. 2313-2319, 2010.

[29] P. G. Kucukkaya, "An exploratory study of positive life changes in Turkish women diagnosed with breast," European Journal of Oncology Nursing, vol. 14, no. 2, pp. 166-173, 2010.

[30] A. W. Wang, C. Chang, S. Chen et al., "Buffering and direct effect of posttraumatic growth in predicting distress following cancer.", Health Psychology, vol. 36, no. 6, pp. 549-559, 2017.

[31] S. C. Danhauer, G. Russell, L. D. Case et al., "Trajectories of Posttraumatic Growth and Associated Characteristics in Women with Breast Cancer," Annals of Behavioral Medicine, vol. 49, no. 5, pp. 650-659, 2015.

[32] M. Barthakur, M. Sharma, S. Chaturvedi, and S. Manjunath, "Posttraumatic growth in women survivors of breast cancer," Indian Journal of Palliative Care, vol. 22, no. 2, p. 157, 2016.

[33] K. Holtmaat, N. van der Spek, P. Cuijpers, C. R. Leemans, and I. M. Verdonck-de Leeuw, "Posttraumatic growth among head and neck cancer survivors with psychological distress," PsychoOncology, vol. 26, no. 1, pp. 96-101, 2017.

[34] N. Koutrouli, F. Anagnostopoulos, and G. Potamianos, "Posttraumatic Stress Disorder and Posttraumatic Growth in Breast Cancer Patients: A Systematic Review," Women \& Health, vol. 52, no. 5, pp. 503-516, 2012.

[35] N. C. Yeung and Q. Lu, "Perceived Stress as a Mediator Between Social Support and Posttraumatic Growth Among Chinese American Breast Cancer Survivors," Cancer Nursing, vol. 41, no. 1, pp. 53-61, 2018.

[36] T. Li, T. Liu, J. Han et al., "The relationship among resilience, rumination and posttraumatic growth in hemodialysis patients in North China," Psychology, Health \& Medicine, vol. 23, no. 4, pp. 442-453, 2017.

[37] D. Nishi, Y. Matsuoka, and Y. Kim, "Posttraumatic growth, posttraumatic stress disorder and resilience of motor vehicle accident survivors," BioPsychoSocial Medicine, vol. 4, 2010.

[38] L. Bolier, M. Haverman, G. J. Westerhof, H. Riper, F. Smit, and E. Bohlmeijer, "Positive psychology interventions: a meta-analysis of randomized controlled studies," BMC Public Health, vol. 13, article 119, 2013.

[39] A. R. Rosenberg, J. P. Yi-Frazier, L. Eaton et al., "Promoting Resilience in Stress Management: A Pilot Study of a Novel Resilience-Promoting Intervention for Adolescents and Young Adults With Serious Illness," Journal of Pediatric Psychology, vol. 40, no. 9, pp. 992-999, 2015.
[40] S. M. Southwick and D. S. Charney, "The Science of Resilience: Implications for the Prevention and Treatment of Depression," Science, vol. 338, no. 6103, pp. 79-82, 2012. 


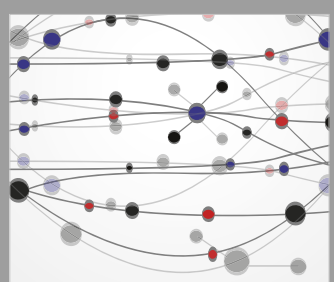

The Scientific World Journal
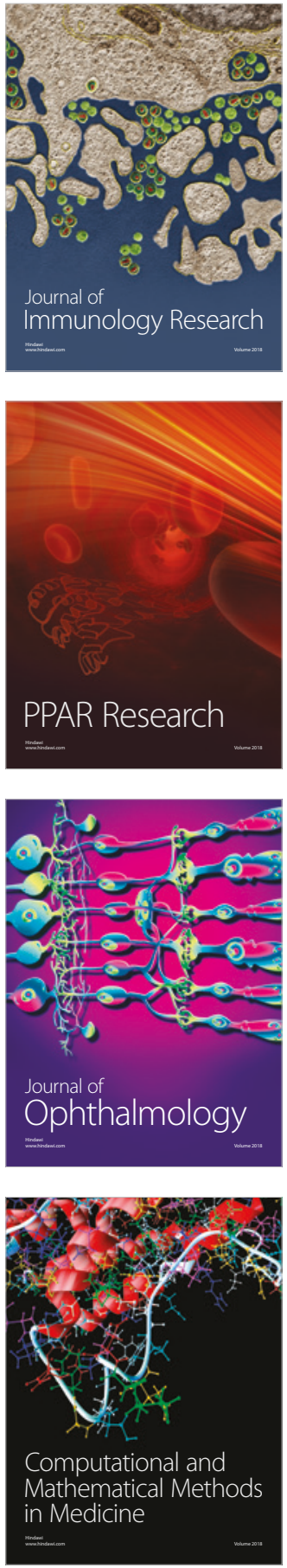

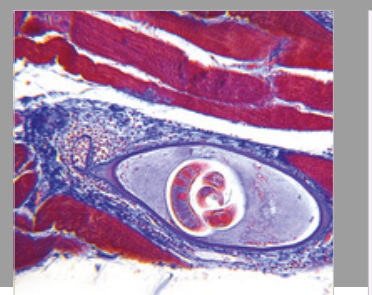

Gastroenterology Research and Practice

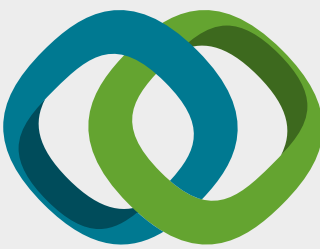

\section{Hindawi}

Submit your manuscripts at

www.hindawi.com
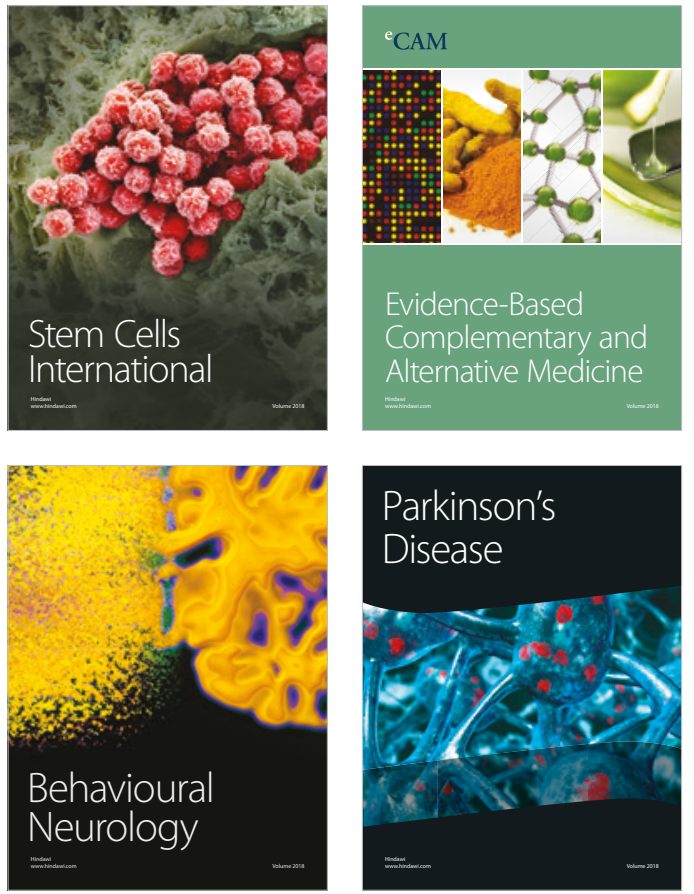

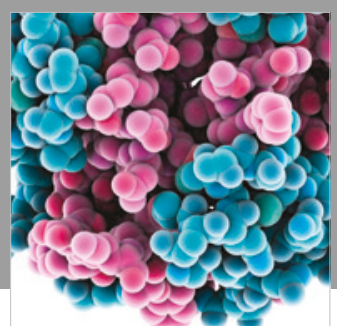

ournal of

Diabetes Research

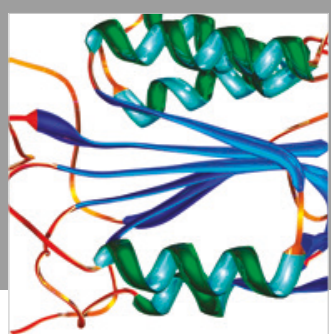

Disease Markers
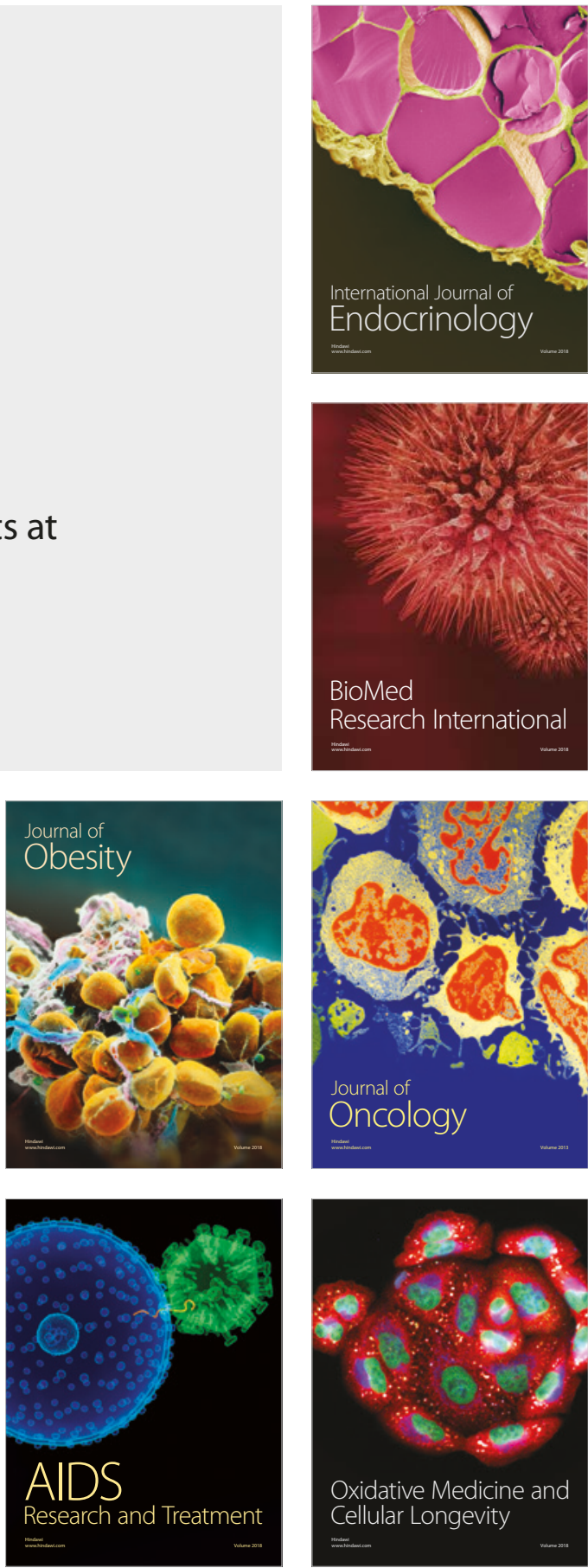